\title{
Prognostic value of lymph node ratio in metastatic papillary thyroid carcinoma
}

\author{
J MANSOUR $^{1}$, D SAGIV $^{1}$, E ALON $^{1,2}$, Y TALMI $^{1,2}$ \\ ${ }^{1}$ Department of Otolaryngology, Head and Neck Surgery, Chaim Sheba Medical Center, Ramat-Gan, and ${ }^{2}$ Sackler \\ Faculty of Medicine, Tel Aviv University, Israel
}

\begin{abstract}
Objective: Cervical metastases in papillary thyroid carcinoma are associated with increased recurrence. However, their effect on survival remains controversial. This study evaluated literature on the prognostic value of lymph node ratio for loco-regional recurrence and survival in metastatic papillary thyroid carcinoma.

Methods: The PubMed database was systematically searched using the terms 'papillary thyroid carcinoma' and 'lymph node ratio'. Articles addressing the association between lymph node ratio and loco-regional recurrence or survival were identified.

Results: Nine retrospective studies were included, comprising 12400 post-thyroidectomy and neck dissection papillary thyroid carcinoma patients (median age, 48.6 years; 76 per cent females). Lymph node ratio was associated with worse recurrence-free survival in 60 and 75 per cent of studies investigating the effect of central compartment metastases and both central and lateral compartment metastases on recurrence-free survival, respectively. One large population-based study showed an association between lymph node ratio and diseasespecific mortality in $\mathrm{N}_{1}$ nodal disease, but failed to maintain the same association when $\mathrm{N}_{1 \mathrm{~b}}$ patients were excluded.

Conclusion: Regional lymph node ratio is an independent predictor for loco-regional recurrence in pathologically staged $\mathrm{N}_{1}$ patients with papillary thyroid carcinoma. Patients with a high lymph node ratio should be closely followed up.
\end{abstract}

Key words: Thyroid Cancer; Carcinoma, Papillary; Lymph Nodes; Neck Dissection; Neoplasm Metastasis

\section{Introduction}

Papillary thyroid carcinoma is the most common form of thyroid malignancy, accounting for $60-70$ per cent of all thyroid cancer, and occurring more often in women in the fourth and fifth decades of life. ${ }^{1}$ Its incidence has more than doubled in the last three decades. ${ }^{2}$ The mortality rate, however, has remained stable with a good prognosis, with a 10-year disease-related survival rate above 90 per cent. ${ }^{1-3}$

Cervical lymph node metastases are found in $20-50$ per cent of cases and in up to 90 per cent when micrometastases are included. Cervical lymph node metastases are considered an independent risk factor for loco-regional recurrence and increased morbidity. ${ }^{4-8}$ Although cervical metastases are clearly associated with an increased recurrence rate, their effect on survival remains controversial. ${ }^{4-8}$ Mazzaferri and Young found that cervical metastases did not influence survival. ${ }^{9}$ However, other population-based studies demonstrated a negative effect on survival, with varying levels of significance. $^{3,10-13}$

The current 2016 American Joint Committee on Cancer tumour-node-metastasis (TNM) staging system for papillary thyroid carcinoma incorporates only the presence and laterality of regional lymph nodes, yet prognosis is affected only in patients older than 45 years. Moreover, it was originally developed to predict risk for mortality rather than recurrence. Although the prognosis of papillary thyroid carcinoma patients with regional disease is quite variable, the American Joint Committee on Cancer TNM staging system does not take into consideration the burden of the metastatic regional disease, and does not differentiate between minimal or extensive metastatic disease in relation to effects on prognosis.

Lymph node ratio is defined as the number of metastatic lymph nodes divided by the number of lymph nodes examined. Lymph node ratio has been shown to be a prognostic variable in non-thyroidal cancers such as head and neck, gastric, breast, and colorectal cancers. ${ }^{14-16}$ In contrast, Roberts et al. demonstrated superior prognostic value for the number of positive lymph nodes, in comparison with lymph node ratio and American Joint Committee on Cancer nodal $(\mathrm{N})$ staging for head and neck cancers. ${ }^{17}$ Multiple recent studies have evaluated the prognostic value of lymph 
node ratio in papillary thyroid carcinoma regarding loco-regional recurrence and survival, with varying results. ${ }^{18-26}$

This study aimed to review recent publications addressing the prognostic value of lymph node ratio in metastatic papillary thyroid carcinoma.

\section{Materials and methods}

A systematic literature review of the PubMed database was conducted on May 2016 using the search terms 'papillary thyroid carcinoma' and 'lymph node ratio'. In addition, we manually searched the references of the identified articles and prior relevant reviews to detect further studies.

All articles that fulfilled our inclusion criteria were included. The studies had to be randomised or nonrandomised controlled trials, or prospective or retrospective studies. The participants were post-thyroidectomy and neck dissection papillary thyroid carcinoma patients. There needed to be data regarding central or lateral cervical lymph nodes and lymph node ratio. Positive lymph nodes were required to be confirmed by pathological examination. Sufficient reported data were required for estimating the prognostic value of lymph node ratio on recurrence and survival.

Where available, the following data were extracted for analysis: number of patients, mean number of lymph nodes retrieved, mean number of metastatic lymph nodes, lymph node ratio, lymph node ratio threshold, hazard ratio, prognosis and survival.

This study was approved by the institutional review board of the Chaim Sheba Medical Center, Ramat-Gan, Israel (reference number: 3142-16-SMC).

\section{Results}

The electronic search primarily yielded 189 articles. Of these, 164 were excluded on the basis of the title or abstract, resulting in 25 potentially eligible studies. After critical examination, nine studies were considered adequate according to the inclusion criteria. ${ }^{18-26}$

These nine studies were published between 2012 and 2016. All studies were retrospective. Overall, 12400 papillary thyroid carcinoma patients were included. All patients had undergone thyroidectomy, and: prophylactic or therapeutic neck dissection; central or lateral compartment neck dissection; or both. Median age at the time of diagnosis was 48.6 years, and 76 per cent of patients were female.

One study utilised the Surveillance, Epidemiology, and End Results database of the National Cancer Institute to evaluate the impact of lymph node ratio in metastatic papillary thyroid carcinoma on diseaserelated mortality. ${ }^{18}$ Eight studies evaluated the prognostic value of lymph node ratio on recurrence-free survival. The patients' characteristics are summarised in Table I. A significant association was found between lymph node ratio and worse recurrence-free survival in 60 per cent and 75 per cent of the studies that investigated the effect of central compartment metastases

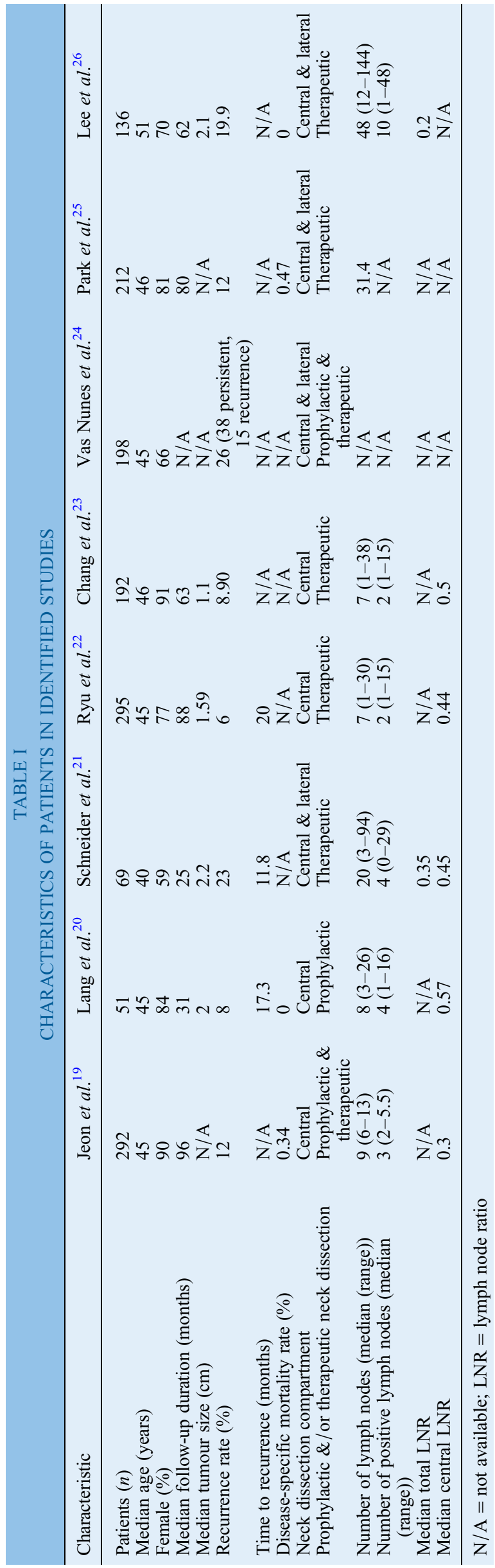


and both central and lateral compartment metastases on recurrence-free survival, respectively. ${ }^{19-26}$ Different lymph node ratio thresholds were used in each study (range, 0.3-0.86). The prognostic values of lymph node ratio and the hazard ratios for loco-regional recurrence are presented in Table II.

\section{Discussion}

Cervical lymph node metastases are found in 20-50 per cent of papillary thyroid carcinoma patients and in up to 90 per cent when including micro-metastases. The current American Joint Committee on Cancer TNM staging system for papillary thyroid carcinoma incorporates only the presence and laterality of regional lymph nodes, and does not take into consideration the burden of metastatic regional disease. Lymph node ratio may have greater prognostic value, as it takes into consideration both the extent of surgery and the regional metastatic burden. Thus, it can differentiate between minimal and extensive metastatic disease, an issue missing from the American Joint Committee on Cancer TNM classification.

Although the data on the extent of nodal involvement and its subsequent effect on disease recurrence and overall survival rates are still debated, previous studies have demonstrated that cervical lymph node metastasis is correlated with loco-regional papillary thyroid carcinoma recurrence. ${ }^{4-8}$ Even though the lymph node ratio has prognostic significance in several non-thyroidal cancers, its significance in papillary thyroid carcinoma remains unclear. ${ }^{14-17}$ This study focused on the regional lymph node ratio in metastatic papillary thyroid carcinoma, and considered its prognostic significance on loco-regional recurrence and survival.

Only one study investigated the effect of lymph node ratio on survival. Schneider et al. utilised a populationlevel database (the Surveillance, Epidemiology, and End Results database) of the National Cancer Institute, and identified 10955 metastatic papillary thyroid carcinoma patients treated with thyroidectomy and neck dissection. ${ }^{18}$ Those authors demonstrated a significant association between lymph node ratio and diseaserelated mortality, with a hazard ratio of 4.33 (95 per cent confidence interval $(\mathrm{CI})=1.68-11.18, p<0.01)$; there was a significant increase in disease-related mortality from 0.65 per cent for patients with a lymph node ratio of less than 0.42 to 1.72 per cent for patients with a lymph node ratio of 0.42 or more $(p<0.01)$. Yet, this association lost its significance when the analyses were performed after excluding patients with $\mathrm{N}_{1 \mathrm{~b}}$ disease, raising the question of whether the significance of lymph node ratio on survival may be attributed to lateral neck disease alone.

\section{Central compartment analysis}

Five out of eight studies ${ }^{19-22,24}$ investigated the effect of central compartment metastases $\left(\mathrm{N}_{1 \mathrm{a}}\right)$ alone on recurrence. Three of the studies ${ }^{19,21,22}$ (60 per cent)

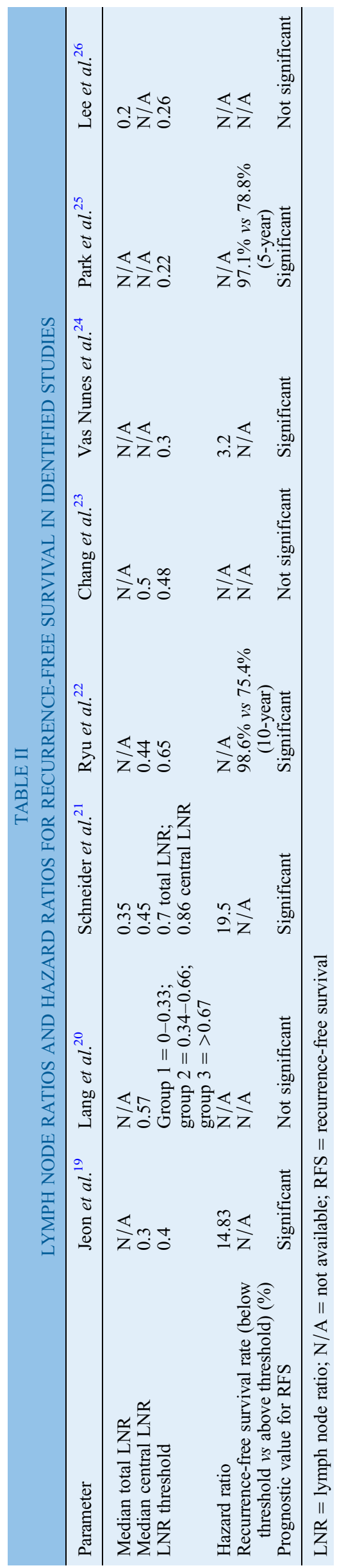


found a significant association between lymph node ratio and recurrence-free survival on multivariate analysis.

Jeon et al. investigated 292 papillary thyroid carcinoma patients who underwent prophylactic (63 per cent) or therapeutic (37 per cent) central neck dissection. ${ }^{19}$ The median numbers of retrieved and metastatic lymph nodes were 9 (range, 6-13) and 3 (range, $2-5.5)$, respectively. The hazard ratio for recurrence in their study population increased significantly from 2.92 (95 per cent $\mathrm{CI}=0.88-9.76, p=0.081$ ) in patients with a lymph node ratio of less than 0.4 to 14.83 (95 per cent $\mathrm{CI}=5.07-43.35, p<0.001$ ) in patients with a lymph node ratio of 0.4 or more. They also found that lymph node ratio had a more distinct effect on prognosis as compared to lymph node size.

Lang et al. conducted their study on 51 clinically staged $\mathrm{N}_{0}$ papillary thyroid carcinoma patients who underwent unilateral prophylactic central neck dissection. ${ }^{20}$ The median numbers of retrieved and metastatic lymph nodes were 8 (range, 3-26) and 4 (range, 1-16), respectively. They found that lymph node ratio was associated with higher post-ablative thyroglobulin levels, which may lead to a greater recurrence rate. However, no direct association between lymph node ratio and recurrence-free survival was found. This is the only study to investigate the prognostic value of lymph node ratio in patients with clinically staged $\mathrm{N}_{0}$ disease.

In a study, by Schneider et al., of 69 patients who underwent therapeutic neck dissection for $\mathrm{N}_{1}$ disease, 35 patients (50.7 per cent) underwent therapeutic central neck dissection for $\mathrm{N}_{1 \mathrm{a}}$ disease. ${ }^{21}$ Only lymph node ratio was significantly associated with recurrence on multivariate analysis, with an average hazard ratio of 19.5 (95 per cent $C I=4.1-22.9, p<0.01$ ) in patients with a central lymph node ratio of 0.86 or more.

Ryu et al. showed that a central lymph node ratio of 0.65 or more was the only independent variable predictive for recurrence in a multivariate analysis of 295 patients with $\mathrm{N}_{1 \text { a }}$ papillary thyroid carcinoma who underwent thyroidectomy and bilateral central neck dissection. ${ }^{22}$ They found no association between recurrence-free survival and tumour size, multi-centricity, thyroiditis and total number of positive lymph nodes. The 10-year estimated recurrence-free survival rates were 98.6 per cent and 75.4 per cent for patients with lymph node ratios of less than 0.65 and 0.65 or more, respectively. The median numbers of retrieved and metastatic lymph nodes were 7 (range, 1-30) and 2 (range, 1-15), respectively.

Chang et al. investigated 192 patients with metastatic papillary thyroid carcinoma who underwent therapeutic central neck dissection. ${ }^{23}$ The median numbers of retrieved and metastatic lymph nodes were 7 (range, 1-38) and 2 (range, 1-15), respectively. Multivariate analysis showed that a lymph node ratio of 0.48 or more was not an independent risk factor for recurrence. Instead, they found that maximal size of metastatic foci, American Thyroid Association risk categories and stimulated serum thyroglobulin levels were predictive of recurrence after surgery. A pre-ablation thyroglobulin level of $9.3 \mathrm{ng} / \mathrm{ml}$ or higher was an independent risk factor for recurrence.

\section{Central and lateral compartment analysis}

Four out of eight studies ${ }^{21,24-26}$ did not differentiate between $\mathrm{N}_{1 \mathrm{a}}$ and $\mathrm{N}_{1 \mathrm{~b}}$ patients when investigating the effect of lymph node ratio on recurrence-free survival. Three of these studies ( 75 per cent) found a significant association between lymph node ratio and recurrencefree survival on multivariate analysis.

As mentioned above, Schneider et al. conducted a study that included 69 patients who underwent therapeutic neck dissection for $\mathrm{N}_{1}$ disease (34 patients underwent central and lateral neck dissection). ${ }^{21}$ The median numbers of retrieved and metastatic lymph nodes were 20 (range, 3-94) and 4 (range, 0-29), respectively. A lymph node ratio of 0.7 or higher was the only significant risk factor for recurrence on multivariate analysis, with a hazard ratio of 19.5 (95 per cent $\mathrm{CI}=4.1-22.9, p<0.01)$. All other prognostic factors, including age, gender, tumour size, multifocality, extrathyroid extension, lymphovascular invasion, extranodal extension and radioactive iodine, failed to predict recurrence on multivariate analysis.

In a study, by Vas Nunes et al., of 198 patients with papillary thyroid carcinoma undergoing prophylactic and therapeutic neck dissection, 60 per cent of the patients had central compartment dissection, and 40 per cent had either lateral compartment dissection or both central and lateral compartment dissection. ${ }^{24}$ Lymph node ratio was associated with a decrease in recurrence-free survival, with a hazard ratio of 3.2 (95 per cent $\mathrm{CI}=1.4-7.3, p=0.005$ ). Patients with a lymph node ratio of 0.3 or more had a 3.4 times higher risk of persistent or recurrent disease compared to patients with a lymph node ratio of 0 , while patients with a lymph node ratio of 0.11 or less had an 80 per cent chance of remaining disease-free over five years.

Park et al. found that loco-regional recurrence-free survival was significantly decreased in patients with more than six metastatic lymph nodes and a lymph node ratio of more than $0.22 .{ }^{25}$ The five-year recurrence-free survival rate for patients with a lymph node ratio of 0.22 or less or a ratio of more than 0.22 was 97.1 per cent and 78.8 per cent, respectively. Their study involved 212 papillary thyroid carcinoma patients with $\mathrm{N}_{1 b}$ disease. The average number of removed lymph nodes per patient was 31.4.

Lee et al. recently published a study on the prognostic value of the lymph node ratio of lateral compartment metastases in papillary thyroid carcinoma on locoregional recurrence. ${ }^{26}$ They failed to show a significant association between lymph node ratio and recurrencefree survival in 136 patients with papillary thyroid carcinoma and clinically staged $\mathrm{N}_{1 b}$ disease who underwent thyroidectomy with therapeutic central and lateral 
neck dissection. However, other prognostic factors, such as largest size of metastatic lymph node, American Thyroid Association risk categories, and stimulated thyroglobulin level, were independent predictive factors of recurrence-free survival on multivariate analysis. The median numbers of dissected and positive lymph nodes were 48 (range, 12-144) and 10 (range, 1-48), respectively.

\section{General discussion}

As papillary thyroid carcinoma is an indolent tumour with an excellent prognosis, it is difficult to adequately evaluate the prognostic significance of lymph node ratio on survival unless data from a large patient population followed for a long period are available for analysis. Only one study investigated the effect of lymph node ratio on disease-specific mortality. ${ }^{18}$

Prophylactic central neck dissection in clinically staged $\mathrm{N}_{0}$ papillary thyroid carcinoma patients is still controversial. Lang et al. showed no association between lymph node ratio and loco-regional recurrence in 51 patients with clinically staged $\mathrm{N}_{0}$ disease. ${ }^{20} \mathrm{~A}$ larger cohort is required to better understand the effect of central compartment micro-metastasis on prognosis.

While the majority of the studies reviewed showed a positive association between lymph node ratio and higher loco-regional recurrence on multivariate analysis, many of these studies failed to show a significant association between recurrence and some of the popular prognostic factors used today, such as the American Joint Committee on Cancer TNM staging system, tumour size, extrathyroid extension, age or gender. This may indicate the need for a new staging system for papillary thyroid carcinoma that incorporates regional metastatic burden in its classification system, to better evaluate recurrence risk in patients with pathologically staged $\mathrm{N}_{1}$ disease. Patients with a higher lymph node ratio should be considered 'high risk' for locoregional recurrence and should be monitored closely during follow up. Early detection of recurrence may lead to effective and overall better management.

We recognise several significant drawbacks in our review. All studies were retrospective, and the majority did not differentiate between therapeutic and prophylactic neck dissections, or between central and lateral compartment analysis. All studies but one included a relatively small number of patients and short followup periods, limiting the studies' ability to assess the relationship between lymph node ratio and diseaserelated survival. Lymph node ratio is affected by the extent of surgery (number of lymph nodes retrieved) and by the enthusiasm of the pathologist, which may greatly alter the result of lymph node ratio. Furthermore, the extent and dosage of radioactive iodine used postoperatively was not included in the multivariate analysis in the majority of the studies.

Future prospective studies examining the relationship of lymph node ratio to recurrence and survival should take into account the laterality of the lymph nodes, their size, and the distinction between microand macro-metastases.

\section{Conclusion}

Regional lymph node ratio in papillary thyroid carcinoma patients with pathologically staged $\mathrm{N}_{1}$ disease was an independent predictor for loco-regional recurrence in 60 per cent and 75 per cent of the studies that investigated the effect of central compartment metastases and both central and lateral compartment metastases on recurrence-free survival, respectively. Many of the well-accepted prognostic factors, such as the American Joint Committee on Cancer TNM classification, extrathyroidal extension, age and gender, failed to predict recurrence on multivariate analysis in many of the studies. A newer classification system that incorporates lymph node ratio as a prognostic factor for recurrence should be considered.

A high lymph node ratio should encourage clinicians to closely follow up metastatic papillary thyroid carcinoma patients. Based on this review, we recommend the close follow up of patients with a lymph node ratio of more than 0.3 in both central and lateral compartment metastases because of the increased risk for recurrence. Lymph node recurrence in the lateral compartment only had an effect on survival. As not all studies reached statistical significance for the association between lymph node ratio and recurrence, large randomised controlled studies should be conducted to better elucidate the prognostic value of the lymph node ratio in metastatic papillary thyroid carcinoma.

\section{References}

1 Shah JP, Loree TR, Dharker D, Strong EW, Begg C, Vlamis V. Prognostic factors in differentiated carcinoma of the thyroid gland. Am J Surg 1992;164:658-61

2 Davies L, Welch HG. Increasing incidence of thyroid cancer in the United States, 1973-2002. JAMA 2006;295:2164-7

3 Hundahl SA, Fleming ID, Fremgen AM, Menck HR. A National Cancer Data Base report on 53,856 cases of thyroid carcinoma treated in the U.S., 1985-1995. Cancer 1998;83:2638-48

4 Qubain SW, Nakano S, Baba M, Takao S, Aikou T. Distribution of lymph node micrometastasis in $\mathrm{pN} 0$ well-differentiated thyroid carcinoma. Surgery 2002;131:249-56

5 Wada N, Suganuma N, Nakayama H, Masudo K, Rino Y, Masuda $\mathrm{M}$ et al. Microscopic regional lymph node status in papillary thyroid carcinoma with and without lymphadenopathy and its relation to outcomes. Langenbecks Arch Surg 2007;392: 417-22

6 Baek SK, Jung KY, Kang SM, Kwon SY, Woo JS, Cho SH et al. Clinical risk factors associated with cervical lymph node recurrence in papillary thyroid carcinoma. Thyroid 2010;20:147-52

7 Sellers M, Beenken S, Blankenship A, Soong SJ, Turbat-Herrera $\mathrm{E}$, Urist $\mathrm{M}$ et al. Prognostic significance of cervical lymph node metastases in differentiated thyroid cancer. Am J Surg 1992;164: 578-81

8 Beasley NJ, Lee J, Eski S, Walfish P, Witterick I, Freeman JL. Impact of nodal metastases on prognosis in patients with well-differentiated thyroid cancer. Arch Otolaryngol Head Neck Surg 2002;128:825-8

9 Mazzaferri EL, Young RL. Papillary thyroid carcinoma: a 10 year follow-up report of the impact of therapy in 576 patients. Am J Med 1981;70:511-18

10 Zaydfudim V, Feurer ID, Griffin MR, Phay JE. The impact of lymph node involvement on survival in patients with papillary and follicular thyroid carcinoma. Surgery 2008;144:1070-7 
11 Scheumann GF, Gimm O, Wegener G, Hundeshagen H, Dralle H. Prognostic significance and surgical management of locoregional lymph node metastases in papillary thyroid cancer. World J Surg 1994;18:559-67

12 Podnos YD, Smith D, Wagman LD, Ellenhorn JD. The implication of lymph node metastasis on survival in patients with welldifferentiated thyroid cancer. Am Surg 2005;71:731-4

13 Ricarte-Filho J, Ganly I, Rivera M, Katabi N, Fu W, Shaha A et al. Papillary thyroid carcinomas with cervical lymph node metastases can be stratified into clinically relevant prognostic categories using oncogenic BRAF, the number of nodal metastases, and extra-nodal extension. Thyroid 2012;22:575-84

14 Ceelen W, Van Nieuwenhove Y, Pattyn P. Prognostic value of the lymph node ratio in stage III colorectal cancer: a systematic review. Ann Surg Oncol 2010;17:2847-55

15 Celen O, Yildirim E, Berberoglu U. Prognostic impact of positive lymph node ratio in gastric carcinoma. J Surg Oncol 2007; 96:95-101

16 Slidell MB, Chang DC, Cameron JL, Wolfgang C, Herman JM, Schulick RD et al. Impact of total lymph node count and lymph node ratio on staging and survival after pancreatectomy for pancreatic adenocarcinoma: a large, population-based analysis. Ann Surg Oncol 2008;15:165-74

17 Roberts TJ, Colevas AD, Hara W, Holsinger FC, Oakley-Girvan I, Divi V. Number of positive nodes is superior to the lymph node ratio and American Joint Committee on Cancer $\mathrm{N}$ staging for the prognosis of surgically treated head and neck squamous cell carcinomas. Cancer 2016;122:1388-97

18 Schneider DF, Chen H, Sippel RS. Impact of lymph node ratio on survival in papillary thyroid cancer. Ann Surg Oncol 2013; 20:1906-11

19 Jeon MJ, Yoon JH, Han JM, Yim JH, Hong SJ, Song DE et al. The prognostic value of the metastatic lymph node ratio and maximal metastatic tumor size in pathological N1a papillary thyroid carcinoma. Eur J Endocrinol 2013;168:219-25

20 Lang BH, Wong KP, Wan KY, Lo CY. Significance of metastatic lymph node ratio on stimulated thyroglobulin levels in papillary thyroid carcinoma after prophylactic unilateral central neck dissection. Ann Surg Oncol 2012;19:1257-63
21 Schneider DF, Mazeh H, Chen H, Sippel RS. Lymph node ratio predicts recurrence in papillary thyroid cancer. Oncologist 2013; 18: $157-62$

22 Ryu IS, Song CI, Choi SH, Roh JL, Nam SY, Kim SY. Lymph node ratio of the central compartment is a significant predictor for locoregional recurrence after prophylactic central neck dissection in patients with thyroid papillary carcinoma. Ann Surg Oncol 2014;21:277-83

23 Chang YW, Kim HS, Jung SP, Kim HY, Lee JB, Bae JW et al. Pre-ablation stimulated thyroglobulin is a better predictor of recurrence in pathological N1a papillary thyroid carcinoma than the lymph node ratio. Int J Clin Oncol 2016;21:862-8

24 Vas Nunes JH, Clark JR, Gao K, Chua E, Campbell P, Niles N et al. Prognostic implications of lymph node yield and lymph node ratio in papillary thyroid carcinoma. Thyroid 2013;23: $811-16$

25 Park YM, Wang SG, Shin DH, Kim IJ, Son SM, Lee BJ. Lymph node status of lateral neck compartment in patients with N1b papillary thyroid carcinoma. Acta Otolaryngol 2016;136: 319-24

26 Lee CW, Roh JL, Gong G, Cho KJ, Choi SH, Nam SY et al. Risk factors for recurrence of papillary thyroid carcinoma with clinically node-positive lateral neck. Ann Surg Oncol 2015;22: $117-24$

Address for correspondence:

Dr Jobran Mansour,

Department of Otolaryngology,

Head and Neck Surgery,

Chaim Sheba Medical Center,

Ramat-Gan 52621, Israel

Fax: +972 (3)530 5124

E-mail: Jobran.h@gmail.com

Dr J Mansour takes responsibility for the integrity of the content of the paper

Competing interests: None declared 